\title{
"Die radiologische Welt ist nicht neu erfunden worden“
}

Radiologische Vorsorgeuntersuchungen und eine Meldepflicht für Dosisüberschreitungen: das neue Strahlenschutzgesetz hält auch einige geänderte Vorgaben für Mediziner bereit. Was RadiologInnen, Medizinisch-Technische RadiologieassistentInnen (MTRA) und MedizinphysikerInnen nun erwartet, erläutern Dipl.-Ing. Horst Lenzen, Leiter der Medizinischen Physik am Universitätsklinikum Münster, und Prof. Dr. Reinhard Loose, ehemaliger Chefarzt am Institut für Radiologie im Klinikum Nürnberg-Nord, im Interview.

DRG: Herr Lenzen, Professor Loose, im Juli wurde offiziell das erste Strahlenschutzgesetz Deutschlands im Bundesgesetzblatt verkündet. Wie viel ändert sich jetzt im radiologischen Berufsalltag?

Dipl.-Ing. Horst Lenzen: Zunächst einmal vorweg: Die radiologische Welt ist nicht neu erfunden worden. Mit wenigen Ausnahmen bleiben die meisten Dinge so wie sie sind. Allerdings wurde die Rechtsnorm für viele Punkte von einer Rechtsverordnung auf ein Bundesgesetz angehoben. Dies signalisiert, dass der Gesetzgeber dem Strahlenschutz künftig eine deutlich höhere Bedeutung zumisst. Verstöße im Rahmen der Medizin werden aber wohl weiterhin als Ordnungswidrigkeit und nicht als Straftatbestand gewertet. Die bisherige Parallelität von Strahlenschutzverordnung und Röntgenverordnung wird abgeschafft und in wichtigen Punkten im neuen Gesetz vereinigt. Ob sich dadurch eine Vereinfachung ergibt, ist zumindest aus Sicht der Radiologie bei 218 Paragrafen in 32 Artikeln fragwürdig. Die Röntgenverordnung war ein übersichtliches Werk, das sich ausschließlich mit der Anwendung von Röntgenstrahlen beschäftigte. Jetzt müssen die für die Medizin bedeutenden Paragrafen zwischen Radon und radioaktiven Altlasten gesucht werden. Noch ist das Gesetz nicht so richtig „scharfgeschaltet“, da wichtige Details über eine Verordnung geregelt werden müssen. Bis dahin gelten die Röntgen- und Strahlen-

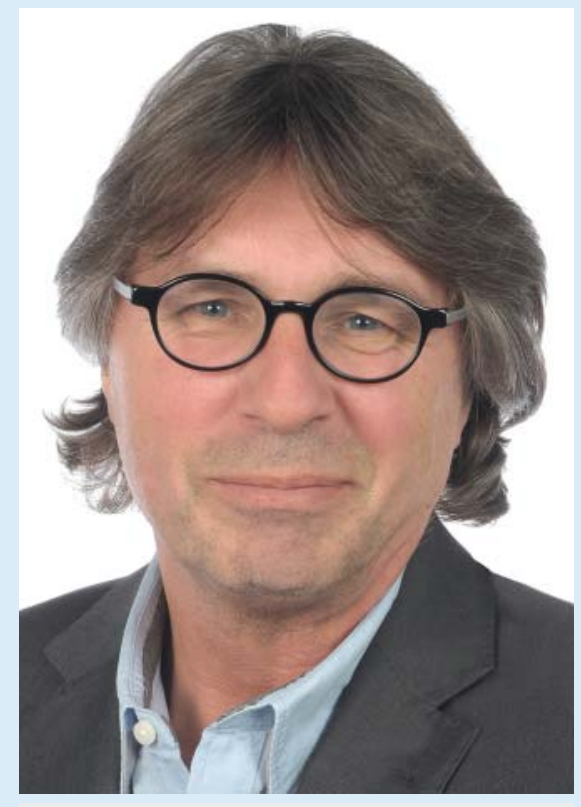

Dipl.-Ing. Horst Lenzen

schutzverordnung fort. Ein gemeinsamer Ersatz ist derzeit in Arbeit und wird bis zum 31.12.2018 in Kraft treten.

Prof. Dr. Reinhard Loose: Wirklich neu ist die Möglichkeit zur Einführung von individuellen Früherkennungsmaßnahmen, die allerdings vorher im Einzelfall vom Bundesamt für Strahlenschutz wissenschaftlich bewertet und vom Umweltministerium freigegeben werden müssen. Im Gespräch sind Früherkennungsuntersuchungen asymptomatischer Personen, also Menschen, die noch keine Patienten sind, mit definierten Risikoprofilen. Hier stehen zur Früherkennung an erster Stelle das CT des Bronchialkarzinoms bei langjährigen Rauchern, gefolgt von der virtuellen CT-Kolonografie zur Erkennung von Darmkrebs und seiner Vorstufen und die CT-Koronar-angiografie zur Erkennung von Erkrankungen der Koronargefäße. Das Ministerium wird die Rahmenbedingungen für die Durchführung festlegen. Ohne ein Genehmigungsverfahren und einen Qualitätsnachweis wird es dabei aber sicher nicht gehen. Vor 2019 ist nicht mit einer ersten Zulassung zu rechnen.

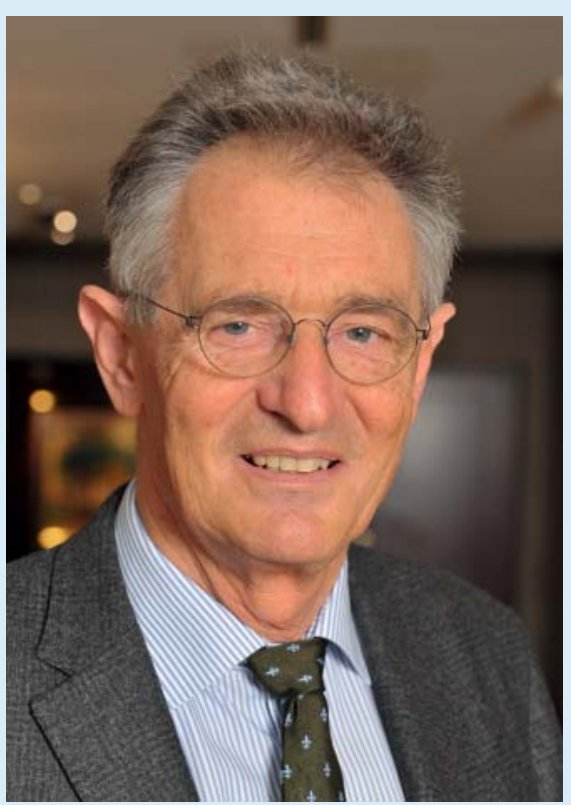

Prof. Dr. Reinhard Loose

Abgesehen von neuen Chancen für die Früherkennung - wird es auch strengere Vorgaben geben?

Lenzen: Erhebliche Dosisüberschreitungen sind zukünftig meldepflichtig. Es ist derzeit noch unklar, ab welcher Dosis und in welcher Form eine Meldepflicht besteht. Je nach Auslegung könnte der Aufwand für die Betreiber nicht unerheblich werden, da die meisten radiologischen Informationssysteme und Röntgenanlagen keine Werkzeuge zur Auswertung bereitstellen. Bevor eine entsprechende Verordnung auf dem Tisch liegt und ein zentrales Meldesystem existiert, sollte man hier allerdings nicht aktiv werden. Im Gesetz heißt es, dass Untersuchungen und Behandlungen mit hohen Dosen nur noch durchgeführt werden dürfen, wenn ein Medizinphysikexperte (MPE) hinzugezogen werden kann. Derzeit kann daraus geschlossen werden, dass dies nur für die Computertomografie und die interventionelle Radiologie gilt. Keinesfalls ist der Begriff „hinzuziehen“ mit der Anwesenheitspflicht für den Medizinphysikexperten in der Strahlentherapie zu verwechseln. Die 
Arbeitsgemeinschaft Physik und Technik der DRG (APT) geht von einem Arbeitsumfang von etwa 15 Manntagen pro Jahr und Gerät aus. Die Aufgaben des MPE liegen unter anderem in der Dosimetrie, der Optimierung von Protokollen, der Berechnung und Überwachung von Expositionen, dem Strahlenschutz des Personals, der Schulung und dem Qualitätsmanagement. Wer den Neukauf eines Gerätes im Jahr 2019 plant, sollte sich frühzeitig um die Zusammenarbeit mit einem Medizinphysikexperten bemühen, da der Markt zurzeit recht eng ist und Neugeräte ab 31.12.2018 nur noch mit MPE in Betrieb genommen werden dürfen.

\section{Was müssen Kliniken und Praxen denn in Bezug auf den viel diskutierten neuen Grenzwert von $\mathbf{2 0}$ mSv für die Augenlinse beachten?}

Lenzen: Die Absenkung von $150 \mathrm{mSv}$ auf $20 \mathrm{mSv}$ wird für die meisten Betriebe kein allzu großes Problem darstellen, zumal derartige Dosen praktisch nur bei interventionellen Maßnahmen vorkommen. Bei konsequentem Einsatz von Bleiglasscheiben und Bleisegeln, möglicherweise kombiniert mit Bleiglasbrillen, lässt sich der Wert gut einhalten. Echtzeitdosimeter können unmittelbar den falschen Einsatz der Strahlenschutzmittel durch eine erhöhte Dosisanzeige während der Maßnahme signalisieren und auf notwendige Korrekturen bei der Positionierung der Strahlenschutzmittel oder der eigenen Position hinweisen.

\section{Gibt es Bereiche, in denen die Umsetzung in der Praxis aus Ihrer Sicht schwieriger wird?}

Lenzen: Schwierigkeiten sehen wir vor allem beim Vollzug durch die Behörden. Wenn durch Personalmangel auf eine Überwachung in vielen Fällen verzichtet werden muss, werden zwangsläufig die schwarzen Schafe geschützt und die Gesetzestreuen bestraft, weil sie mit viel Mühen und finanziellem Einsatz die Vorgaben umsetzen. Hierdurch könnte sich schnell Unmut bei alen Beteiligten regen. Zumal den Behörden weiterer Personalbe- darf ins Haus steht, da das Gesetz von den Behörden die regelmäßige Durchführung risikoorientierter Überwachungsprogramme fordert. Das Antragsverfahren für die Forschung wurde in vielen Punkten verändert. So wurde das bisherige „vereinfachte Verfahren“, zum Beispiel für die Arzneimittelprüfung, in ein Anzeigeverfahren mit einer maximalen Bearbeitungszeit von 28 Tagen verändert. Dies bedeutet, dass die Behörde jetzt lediglich ein Einspruchsrecht hat und nach Fristablauf das Projekt durchgeführt werden kann. Projekte, die die Anwendung der Strahlung als Hauptziel verfolgen, sind weiterhin genehmigungspflichtig. Allerding muss die Behörde jetzt das Verfahren nach 90 Tagen abschließen. Dies ist ein Schritt in die richtige Richtung, da die bisher langen Wartezeiten Forschungsvorhaben oft behindert haben. Wie häufig sich trotzdem die Antragszeit auf 180 Tage verdoppelt, wenn die Behörde von der Ausnahmeregelung wegen Personalmangels Gebrauch macht, bleibt abzuwarten. Spätestens dann wäre man für die Radiologie wieder bei den alten Verhältnissen.

\section{Ändern sich auch die Anforderungen an FachärztInnen, MPE und MTRA für den Sach- und Fachkundeerwerb sowie für die Aktualisierung?}

Lenzen: Im Moment ist davon auszugehen, dass die Inhalte der Fachkunderichtlinie bestehen bleiben und lediglich aus der Richtlinie in eine juristisch sichere Verwaltungsvorschrift überführt werden. Das Bundesministerium für Umwelt, Naturschutz, Bau und Reaktorsicherheit (BMUB) plant jedoch ab 2019 eine Anpassung der bisherigen Fachkunderichtlinien. Für den MPE wird es wegen seiner neuen Aufgaben aber vermutlich einige Änderungen geben. Auch für die Kurse könnten Anpassungen notwendig werden.

Loose: Viele Radiologen haben auch die Hoffnung, dass es eine Erleichterung in der Teleradiologie geben wird. Die bisherige Forderung der „Gesamtfachkunde“ des Teleradiologen wurde fallen gelassen und durch „erforderliche Fachkunde“ ersetzt.
Wie diese genau aussieht und weitere Rahmenbedingungen werden erst in der Verordnung beschrieben, doch steht bereits jetzt im Gesetz, dass eine regelmäßige und enge Einbindung des Teleradiologen in den klinischen Betrieb des Strahlenschutzverantwortlichen gewährleistet sein muss.

Sie sprachen an, dass wichtige Details später geregelt werden - gilt das Gesetz dann trotzdem ab sofort? Oder gibt es Übergangsfristen?

Lenzen: Sofort wirksam wird lediglich die Aufhebung des Strahlenschutzvorsorgegesetzes, das 1986 in Folge des Reaktorunfalls von Tschernobyl zur Überwachung der Kontaminationen der Umwelt eingerichtet wurde. Die neuen Regelungen sind jetzt Bestandteil des Strahlenschutzgesetzes.

Übergangsvorschriften werden in 22 Artikeln beschrieben und zeigen, dass viele Änderungen zwar bereits am 1.10.2017 in Kraft treten. Die wichtigsten Änderungen für die Medizin greifen allerdings erst ab dem 31.12.2018, etwa der Medizinphysikexperte für Neugeräte. Für Altgeräte ist ein MPE erst nach dem 31.12.2022 Pflicht. Der Grenzwert für die Augenlinse gilt ab dem 31.12.2018. Es sollte allerdings bedacht werden, dass der Grenzwert seit vielen Jahren in Berichten der International Commission on Radiological Protection (ICRP) und EU-Richtlinien beschrieben wird. Schäden bei Arbeitnehmern, die vor dem Übergangstermin unter erhöhten Werten gearbeitet haben, könnten also möglicherweise als Missachtung des aktuellen Standes von Wissenschaft und Technik gewertet werden und damit trotzdem Haftungsansprüche nach sich ziehen. Man wird also in den nächsten Jahren aufmerksam verfolgen müssen, was sich jeweils ändert. Die APT wird auf die jeweiligen Termine frühzeitig hinweisen. Und sicher werden wir die Auslegungen der jeweils anstehenden Änderungen im Forum Röntgenverordnung (hier wird sich der Name ändern müssen) auf dem Deutschen Röntgenkongress eifrig diskutieren.

Herr Lenzen, Professor Loose, vielen Dank für das Gespräch! 